J. Korean Math. Soc. 49 (2012), No. 5, pp. 881-891

http://dx.doi.org/10.4134/JKMS.2012.49.5.881

\title{
CONTROLLABILITY FOR NONLINEAR VARIATIONAL EVOLUTION INEQUALITIES
}

\author{
Jong Yeoul Park, Jin-Mun Jeong, and Hyun-Hee Rho
}

\begin{abstract}
In this paper we investigate the approximate controllability for the following nonlinear functional differential control problem:

$$
x^{\prime}(t)+A x(t)+\partial \phi(x(t)) \ni f(t, x(t))+h(t)
$$
\end{abstract}

which is governed by the variational inequality problem with nonlinear terms.

\section{Introduction}

Let $H$ and $V$ be two complex Hilbert spaces. Assume that $V$ is a dense subspace in $H$ and the injection of $V$ into $H$ is continuous. The norm on $V$ (resp. $H$ ) will be denoted by $\|\cdot\|(\operatorname{resp} .|\cdot|)$. Let $U$ be a complex Banach space and $B$ be a bounded linear operator from $L^{2}(0, T ; U)$ to $L^{2}(0, T ; H)$. Let $A$ be a continuous linear operator from $V$ into $V^{*}$ and satisfies the coercive condition, $\phi: V \rightarrow(-\infty,+\infty]$ be a lower semicontinuous, proper convex function. Then we deal with the approximate controllability for the following control system governed by the variational inequality problem with nonlinear term:

$$
\left\{\begin{aligned}
&\left(x^{\prime}(t)+A x(t), x(t)-z\right)+\phi(x(t))-\phi(z) \\
& \leq(f(t, x(t))+(B u)(t), x(t)-z), \text { a.e., } 0<t \leq T, \quad z \in V, \\
& x(0)=x_{0} .
\end{aligned}\right.
$$

Noting that the subdifferential operator $\partial \phi: V \rightarrow V^{*}$ of $\phi$ is defined by

$$
\partial \phi(x)=\left\{x^{*} \in V^{*} ; \phi(x) \leq \phi(y)+\left(x^{*}, x-y\right), \quad y \in V\right\},
$$

where $(\cdot, \cdot)$ denotes the duality pairing between $V^{*}$ and $V$, the control system $(\mathrm{SE})$ is represented by the following nonlinear functional differential control

Received March 10, 2010; Revised January 2, 2012.

2010 Mathematics Subject Classification. Primary 49J40, 93C20.

Key words and phrases. approximate controllability, variational inequality, subdifferential operator, degree theory.

This research was supported by Basic Science Research Program through the National research Foundation of Korea(NRF) funded by the Ministry of Education, Science and Technology(2011-0026609). 
problem on $H$

$$
\left\{\begin{array}{l}
x^{\prime}(t)+A x(t)+\partial \phi(x(t)) \ni f(t, x(t))+B u(t), \quad 0<t \leq T, \\
x(0)=x_{0},
\end{array}\right.
$$

where the nonlinear mapping $f$ is a Lipschitz continuous from $\mathbb{R} \times V$ into $H$. Its corresponding linear variational inequality $[f \equiv 0$ in $(\mathrm{SE})]$ was widely developed as seen in Section 4.3.2 of Barbu [3] (also see Section 4.3.1 in [2], $[4,5,8,10])$. In [6], using more general hypotheses for nonlinear term $f(\cdot, x)$, we investigated the existence and the norm estimate of a solution of the above nonlinear equation on $L^{2}(0, T ; V) \cap W^{1,2}\left(0, T ; V^{*}\right)$ considered as an equation in $H$ as well as in $V^{*}$, which is also applicable to optimal control problem.

In the paper [9] Naito investigated the semilinear parabolic evolution equation in a Hilbert space $H$, in case where the nonlinear term $f$ is a bounded uniformly continuous mapping from $H$ to itself, and proved the approximate controllability under the following hypothesis;

(B) For each $p \in L^{2}(0, T ; X)$ there exists a function $q \in \bar{X}_{B}: \tilde{S} p=\tilde{S} q$, where

$$
\tilde{S} p=\int_{0}^{T} S(T-s) p(s) d s
$$

$S(\cdot)$ is the semigroup generated by $-A$ and $\bar{X}_{B}$ is the closure in $L^{2}(0, T ; X)$ of the range $X_{B}$ of the operator $B$.

The purpose of this paper is to show that the reachable set of the nonlinear variational inequality (SE) is equivalent to that of its corresponding linear variational inequality under the hypothesis (B) by applying results of [9] to the equation (NCE). We formulate our nonlinear variational evolution inequality (NCE) as a semilinear control system in order to obtain the control problems. As in $[7,9]$ we must assume the uniform boundedness of the nonlinear terms $f(t, x)$ and $(\partial \phi)^{0}$, where $(\partial \phi)^{0}: H \rightarrow H$ is the minimum element of $\partial \phi$. Since we apply the degree of mapping theorem in the proof of the main theorem, we need some compactness hypothesis. We make the natural assumption that the embedding $D\left(A_{0}\right) \subset V$ is compact. Then the embedding $L^{2}\left(0, T ; D\left(A_{0}\right)\right) \cap$ $W^{1,2}(0, T ; H) \subset L^{2}(0, T ; V)$ is compact in view of Aubin's result [1] (see also $[11]$ ), and we show that the mapping which maps $f$ to the solution of (NCE) with $B u$ replaced by $f$ is a compact operator from $L^{2}(0, T ; H)$ to itself. We show the approximate controllability of (NCE) by using the Lelay-Schauder degree theory.

\section{Variational inequalities}

Let $V$ and $H$ be complex Hilbert spaces forming Gelfand triple $V \subset H \subset V^{*}$ with pivot space $H$. For the sake of simplicity, we may consider

$$
\|u\|_{*} \leq|u| \leq\|u\|, \quad u \in V
$$


where $\|\cdot\|_{*}$ is the norm of the element of $V^{*}$. If an operator $A$ is bounded linear from $V$ to $V^{*}$ and generates an analytic semigroup, then it is easily seen that

$$
H=\left\{x \in V^{*}: \int_{0}^{T}\left\|A e^{t A} x\right\|_{*}^{2} d t<\infty\right\}
$$

for the time $T>0$. Therefore, in terms of the intermediate theory we can see that

$$
\left(V, V^{*}\right)_{1 / 2,2}=H \text {. }
$$

We also assume that there exists a constant $C_{1}$ such that

$$
\|u\| \leq C_{1}\|u\|_{D(A)}^{1 / 2}|u|^{1 / 2}
$$

for every $u \in D\left(A_{0}\right)$, where

$$
\|u\|_{D(A)}=\left(|A u|^{2}+|u|^{2}\right)^{1 / 2}
$$

is the graph norm of $D(A)$. Thus, in what follows we will write

$$
V=(D(A), H)_{1 / 2,2}
$$

as a matter of convenience. Let $a(\cdot, \cdot)$ be a bounded sesquilinear form defined in $V \times V$ and satisfying Gårding's inequality

$$
\text { Re } a(u, u) \geq c_{0}\|u\|^{2}, \quad c_{0}>0 .
$$

Let $A$ be the operator associated with the sesquilinear form $a(\cdot, \cdot)$ :

$$
(A u, v)=a(u, v), \quad u, v \in V .
$$

Then $A$ is a bounded linear operator from $V$ to $V^{*}$, and $A$ generates an analytic semigroup in both of $H$ and $V^{*}$. It is also known that if $a(\cdot, \cdot)$ is a symmetric quadratic form satisfying (2.2), then $A$ is positive definite and self-adjoint and $D\left(A^{1 / 2}\right)=V$.

Let $\phi: V \rightarrow(-\infty,+\infty]$ be a lower semicontinuous, proper convex function. Then the subdifferential operator $\partial \phi: V \rightarrow V^{*}$ of $\phi$ is defined by

$$
\partial \phi(x)=\left\{x^{*} \in V^{*} ; \phi(x) \leq \phi(y)+\left(x^{*}, x-y\right), \quad y \in V\right\} .
$$

First, let us consider the following perturbation of subdifferential operator;

$$
\left\{\begin{array}{l}
x^{\prime}(t)+A x(t)+\partial \phi(x(t)) \ni B u(t), \quad 0<t \leq T, \\
x(0)=x_{0} .
\end{array}\right.
$$

For every $\epsilon>0$, define

$$
\phi_{\epsilon}(x)=\inf \left\{\left\|x-J_{\epsilon} x\right\|_{*}^{2} / 2 \epsilon+\phi\left(J_{\epsilon} x\right): x \in V\right\},
$$

where $J_{\epsilon}=(I+\epsilon \partial \phi)^{-1}$. If $B=\partial \phi$, then the function $\partial \phi_{\epsilon}$ is Fréchet differentiable on $V$ and its Frećhet differential $\partial \phi_{\epsilon}=B_{\epsilon}$ is Lipschitz continuous on $H$ with Lipschitz constant $\epsilon^{-1}$ where $B_{\epsilon}=\epsilon^{-1}\left(I-(I+\epsilon B)^{-1}\right)$ is as seen in Corollary 2.2 in Chapter II of [2]. It is also well known results that $\lim _{\epsilon \rightarrow 0} \phi_{\epsilon}=\phi$ and $\lim _{\epsilon \rightarrow 0} \partial \phi_{\epsilon}(x)=(\partial \phi)^{0}(x)$ for every $x \in D(\partial \phi)$ where $(\partial \phi)^{0}: V \rightarrow V^{*}$ is 
the minimum element of $\partial \phi$. Now, we introduce the smoothing system corresponding to $(\mathrm{NE})$ as follows.

$$
\left\{\begin{array}{l}
x^{\prime}(t)+A x(t)+\partial \phi_{\epsilon}(x(t))=B u(t), \quad 0<t \leq T, \\
x(0)=x_{0} .
\end{array}\right.
$$

Using the regularity for the abstract linear parabolic equation we have the following result on the equation (NE).

Proposition 2.1. 1) Let $u \in L^{2}(0, T ; U)$ and $x_{0} \in V$ satisfying that $\phi\left(x_{0}\right)<$ $\infty$. Then the equation (NE) has a unique solution

$$
x \in L^{2}(0, T ; V) \cap W^{1,2}\left(0, T: V^{*}\right) \subset C([0, T] ; H),
$$

which satisfies

$$
x^{\prime}(t)=B u(t)-A x(t)-\partial \phi^{0}(x(t))
$$

and

$$
\|x\|_{L^{2} \cap W^{1,2}} \leq C_{2}\left(1+\left\|x_{0}\right\|+\|u\|_{L^{2}(0, T ; U)}\right),
$$

where $C_{2}$ is a constant and $L^{2} \cap W^{1,2}=L^{2}(0, T ; V) \cap W^{1,2}\left(0, T ; V^{*}\right)$.

2) Let $a(\cdot, \cdot)$ be a symmetric quadratic form satisfying (2.2) and the following hypothesis hold:

(A) There exists $h \in H$ such that for every $\epsilon>0$ and an $\in D(\phi)$

$$
J_{\epsilon}(y+\epsilon h) \in D(\phi) \text { and } \phi\left(J_{\epsilon}(y+\epsilon h)\right) \leq \phi(y) .
$$

Then for $u \in L^{2}(0, T ; U)$ and $x_{0} \in \overline{D(\phi)} \cap V$ the equation (NE) has a unique solution

$$
x \in L^{2}(0, T ; D(A)) \cap W^{1,2}(0, T ; H) \cap C([0, T] ; H),
$$

which satisfies

$$
\|x\|_{L^{2} \cap W^{1,2} \cap C} \leq C_{2}\left(1+\left\|x_{0}\right\|+\|u\|_{L^{2}(0, T ; U)}\right) .
$$

If $V$ is compactly embedded in $H$, the following embedding

$$
\left.L^{2}(0, T ; V) \cap W^{1,2}\left(0, T ; V^{*}\right) \subset L^{2}(0, T ; H)\right)
$$

is compact in view of Theorem 2 of Aubin [1]. Hence, the mapping $u \mapsto x$ is compact from $L^{2}(0, T ; U)$ to $L^{2}(0, T ; H)$.

Now we give the assumption on the nonlinear terms as follows. that

(F) Let $f$ be a nonlinear single valued mapping from $V$ into $H$. We assume

$$
\left|f\left(t, x_{1}\right)-f\left(t, x_{2}\right)\right| \leq L|| x_{1}-x_{2} \|
$$

for every $x_{1}, x_{2} \in V$.

Now, we introduce smoothing system corresponding to (NCE) as follows.

$$
\left\{\begin{array}{l}
\frac{d x(t)}{d t}+A x(t)+\partial \phi_{\epsilon}(x(t))=f(t, x(t))+B u(t), \quad 0<t \leq T, \\
x(0)=x_{0} .
\end{array}\right.
$$


Since $-A$ generates a semigroup $S(t)$ on $H$, the mild solution of (SCE) can be represented by

$$
x_{\epsilon}(t)=S(t) x_{0}+\int_{0}^{t} S(t-s)\left\{f\left(s, x_{\epsilon}(s)\right)+B u(s)-\partial \phi_{\epsilon}\left(x_{\epsilon}(s)\right)\right\} d s .
$$

We establish the following result on the solvability of (NCE) as is seen in Theorem 2.1 of $[6]$.

Proposition 2.2. 1) Let $x_{0} \in V$ satisfying that $\phi\left(x_{0}\right)<\infty, u \in L^{2}(0, T ; U)$ and the assumption (F) be satisfied. Then the equation (NCE) has a unique solution

$$
x \in L^{2}(0, T ; V) \cap W^{1,2}(0, T ; V) \cap C([0, T] ; H),
$$

which satisfies

$$
x^{\prime}(t)=f(t, x(t))+B u(t)-A x(t)-\partial \phi^{0}(x(t))
$$

and there exists a constant $C_{3}$ depending on $T$ such that

$$
\|x\|_{L^{2} \cap W^{1,2}} \leq C_{3}\left(1+\left\|x_{0}\right\|+\|u\|_{L^{2}(0, T ; U)}\right) .
$$

2) Let $a(\cdot, \cdot)$ be a symmetric quadratic form satisfying $(2.2)$ and let us assume the hypotheses $(\mathrm{A}),(\mathrm{F})$. Then the equation $(\mathrm{NCE})$ has a unique solution

$$
x \in L^{2}(0, T ; D(A)) \cap W^{1,2}(0, T ; H) \cap C([0, T] ; H),
$$

which satisfies

$$
\|x\|_{L^{2} \cap W^{1,2} \cap C} \leq C_{3}\left(1+\left\|x_{0}\right\|+\|u\|_{L^{2}(0, T ; U)}\right) .
$$

Theorem 2.3. Let $x_{0} \in V, u \in L^{2}(0, T ; U)$ and the hypotheses in 2$)$ of Proposition 2.2 be satisfied. Then the solution $x$ of the equation (SCE) belongs to $L^{2}(0, T ; D(A)) \cap W^{1,2}(0, T ; H)$, and the mapping

$$
V \times L^{2}(0, T ; U) \ni\left(x_{0}, u\right) \mapsto x \in L^{2}(0, T ; D(A)) \cap W^{1,2}(0, T ; H)
$$

is continuous.

Proof. Let $\left(x_{0 i}, u_{i}\right) \in F \times L^{2}(0, T ; U)$, and $x_{i}$ be the solution of (SNE) with $\left(x_{0 i}, u_{i}\right)$ in place of $\left(x_{0}, u\right)$ for $i=1,2$. Then in view of $(2.7)$, we have

$$
\begin{aligned}
& \left\|x_{1}-x_{2}\right\|_{L^{2}(0, T ; D(A)) \cap W^{1,2}(0, T ; H)} \\
\leq & C_{3}\left\{\left\|x_{01}-x_{02}\right\|+\left(\left\|\partial \phi_{\epsilon}\left(x_{1}\right)-\partial \phi_{\epsilon}\left(x_{2}\right)\right\|+\left\|f\left(\cdot, x_{1}\right)-f\left(\cdot, x_{2}\right)\right\|\right)_{L^{2}(0, T ; H)}\right. \\
& \left.\left.+\left\|u_{1}-u_{2}\right\|\right)_{L^{2}(0, T ; U)}\right\} \\
\leq & C_{3}\left\{\left\|x_{01}-x_{02}\right\|+\left(\epsilon^{-1}+L\right)\left\|x_{1}-x_{2}\right\|_{L^{2}(0, T: V)}+\left\|u_{1}-u_{2}\right\|_{L^{2}(0, T ; U)}\right\} .
\end{aligned}
$$

Noting that

$$
x_{1}(t)-x_{2}(t)=x_{01}-x_{02}+\int_{0}^{t}\left(\dot{x}_{1}(s)-\dot{x}_{2}(s)\right) d s,
$$


we get

$$
\left\|x_{1}-x_{2}\right\|_{L^{2}(0, T ; H)} \leq \sqrt{T}\left\|x_{01}-x_{02}\right\|+\frac{T}{\sqrt{2}}\left\|x_{1}-x_{2}\right\|_{W^{1,2}(0, T ; H)} .
$$

Hence from (2.1) we get

$$
\begin{aligned}
& \left\|x_{1}-x_{2}\right\|_{L^{2}(0, T ; V)} \\
\leq & C_{1}\left\|x_{1}-x_{2}\right\|_{L^{2}(0, T ; D(A))}^{1 / 2}\left\|x_{1}-x_{2}\right\|_{L^{2}(0, T ; H)}^{1 / 2} \\
\leq & C_{1}\left\|x_{1}-x_{2}\right\|_{L^{2}\left(0, T ; D\left(A_{0}\right)\right)}^{1 / 2} \\
& \times\left\{T^{1 / 4}\left\|x_{01}-x_{02}\right\|^{1 / 2}+\left(\frac{T}{\sqrt{2}}\right)^{1 / 2}\left\|x_{1}-x_{2}\right\|_{W^{1,2}(0, T ; H)}^{1 / 2}\right\} \\
\leq & C_{1} T^{1 / 4}\left\|x_{01}-x_{02}\right\|^{1 / 2}\left\|x_{1}-x_{2}\right\|_{L^{2}(0, T ; D(A))}^{1 / 2} \\
& +C_{1}\left(\frac{T}{\sqrt{2}}\right)^{1 / 2}\left\|x_{1}-x_{2}\right\| L_{L^{2}(0, T ; D(A)) \cap W^{1,2}(0, T ; H)} \\
\leq & 2^{-7 / 4} C_{1}\left\|x_{01}-x_{02}\right\|+2 C_{1}\left(\frac{T}{\sqrt{2}}\right)^{1 / 2}\left\|x_{1}-x_{2}\right\|_{L^{2}(0, T ; D(A)) \cap W^{1,2}(0, T ; H)} .
\end{aligned}
$$

Combining (2.8) and (2.9) we obtain

$$
\begin{aligned}
& \left\|x_{1}-x_{2}\right\|_{L^{2}(0, T ; D(A)) \cap W^{1,2}(0, T ; H)} \\
\leq & C_{3}\left\{\left\|x_{01}-x_{02}\right\|+\left(\epsilon^{-1}+L\right)\left(2^{-7 / 4} C_{1}\left\|x_{01}-x_{02}\right\|\right.\right. \\
& \left.\left.+2 C_{1}\left(\frac{T}{\sqrt{2}}\right)^{1 / 2}\left\|x_{1}-x_{2}\right\|_{L^{2}(0, T ; D(A)) \cap W^{1,2}(0, T ; H)}\right)+\left\|u_{1}-u_{2}\right\|_{L^{2}(0, T ; U)}\right\} .
\end{aligned}
$$

Suppose that $\left(x_{0 n}, u_{n}\right) \rightarrow\left(x_{0}, u\right)$ in $V \times L^{2}(0, T ; H)$, and let $x_{n}$ and $x$ be the solutions (SCE) with $\left(x_{0 n}, u_{n}\right)$ and $\left(x_{0}, u\right)$, respectively. Let $0<T_{1} \leq T$ be such that

$$
\left(\epsilon^{-1}+L\right) C_{1} C_{3}\left(2 T_{1}\right)^{1 / 2}<1 .
$$

Then by virtue of (2.10) with $T$ replaced by $T_{1}$ we see that $x_{n} \rightarrow x$ in $L^{2}\left(0, T_{1} ; D(A)\right) \cap W^{1,2}\left(0, T_{1} ; H\right) \subset C\left(\left[0, T_{1}\right] ; V\right)$. This implies that $x_{n}\left(T_{1}\right)$ $\mapsto x\left(T_{1}\right)$ in $V$. Hence the same argument shows that $x_{n} \rightarrow x$ in

$$
L^{2}\left(T_{1}, \min \left\{2 T_{1}, T\right\} ; D(A)\right) \cap W^{1,2}\left(T_{1}, \min \left\{2 T_{1}, T\right\} ; H\right) .
$$

Repeating this process we conclude that

$$
x_{n} \rightarrow x \text { in } L^{2}(0, T ; D(A)) \cap W^{1,2}(0, T ; H) .
$$

\section{Approximate controllability}

In this section we show the approximate controllability for the equation (NCE), which is the extended result of Naito [9] to the equation (SCE). The realization for the operator $A$ in $H$ which is the restriction of $A$ to

$$
D(A)=\{u \in V ; A u \in H\}
$$

be also denoted by $A$. 
The solutions of (NCE) and (SCE) are denoted by $x(t ; \phi, f, u)$ and $x_{\epsilon}\left(t ; \phi_{\epsilon}, f\right.$, $u$ ), respectively. In view of Proposition 2.2, we have

$$
\left\|x_{\epsilon}\left(\cdot ; \phi_{\epsilon}, g, u\right)\right\|_{L^{2}(-h, T ; V) \cap W^{1,2}\left(0, T ; V^{*}\right)} \leq C_{3}\left(1+\left\|x_{0}\right\|+\|u\|_{L^{2}(0, T ; U)}\right) .
$$

For $k \in L^{2}(0, T ; H)$ let $y_{k}$ be the solution of equation with $B=I$

$$
\left\{\begin{array}{l}
x^{\prime}(t)+A x(t)+\partial \phi_{\epsilon}(x(t))=f(x(t))+k(t), \quad 0<t \leq T, \\
x(0)=0 .
\end{array}\right.
$$

Lemma 3.1. Let $k \in L^{2}(0, T ; H)$ and the hypotheses in 2$)$ of Proposition 2.2 be satisfied. Then the solution $y_{k}$ of the equation (3.2) belongs to $L^{2}(0, T ; D(A))$ $\cap W^{1,2}(0, T ; H)$, and the mapping $k \mapsto y_{k}$ is compact from $L^{2}(0, T ; H)$ to $L^{2}(0, T ; V)$.

Proof. From Proposition 2.2 we have that

$$
y_{k}(t)=\int_{0}^{t} S(t-s)\left\{f\left(x_{\epsilon}(s)\right)+k(s)-\partial \phi_{\epsilon}\left(x_{\epsilon}(s)\right)\right\} d s
$$

and

$$
\left\|y_{k}\right\|_{L^{2}\left(0, T ; D\left(A_{0}\right)\right) \cap W^{1,2}(0, T ; H)} \leq C_{3}\left(1+\|k\|_{L^{2}(0, T ; H)}\right) .
$$

Hence if $k$ is bounded in $L^{2}(0, T ; H)$, then so is $y_{k}$ in $L^{2}\left(0, T ; D\left(A_{0}\right)\right) \cap W^{1,2}(0$, $T ; H)$. Since $D\left(A_{0}\right)$ is compactly embedded in $V$ by assumption, the embedding

$$
\left.L^{2}\left(0, T ; D\left(A_{0}\right)\right) \cap W^{1,2}(0, T ; H) \subset L^{2}(0, T ; V)\right)
$$

is compact in view of Theorem 2 of J. P. Aubin [1].

For the sake of simplicity we assume that $S(t)$ is uniformly bounded; there exists a constant $M \geq 1$ such that

$$
\|S(t)\| \leq M \text {. }
$$

To prove the approximate controllability for the equation (NCE) we need the hypothesis that

(A1) $(\partial \phi)^{0}$ is uniformly bounded, i.e.,

$$
\left|(\partial \phi)^{0} x\right| \leq M_{1}, \quad x \in H,
$$

where $(\partial \phi)^{0}: H \rightarrow H$ is the minimum element of $\partial \phi$.

Lemma 3.2. Let the assumption (A1) be satisfied. Then there exists a constant $C$ independent of $\epsilon$ such that

$$
\left\|x_{\epsilon}-x\right\|_{C([0, T] ; H) \cap L^{2}(0, T ; V)} \leq C \epsilon, \quad 0<T .
$$

Proof. For any $\epsilon>0$ and $\lambda>0$, let $x_{\epsilon}$ and $x_{\lambda}$ be the solutions of (SCE) corresponding to $\epsilon$ and $\lambda$, respectively. Then from the equation (SCE) we have

$$
\begin{aligned}
& x_{\epsilon}^{\prime}(t)-x_{\lambda}^{\prime}(t)+A\left(x_{\epsilon}(t)-x_{\lambda}(t)\right)+\partial \phi_{\epsilon}\left(x_{\epsilon}(t)\right)-\partial \phi_{\lambda}\left(x_{\lambda}(t)\right) \\
= & f\left(x_{\epsilon}(t)\right)-f\left(x_{\lambda}(t)\right),
\end{aligned}
$$


and hence, from (2.2) and multiplying by $x_{\epsilon}-x_{\lambda}(t)$, it follows that

$$
\begin{aligned}
& \frac{1}{2} \frac{d}{d t}\left|x_{\epsilon}(t)-x_{\lambda}(t)\right|^{2}+c_{0}|| x_{\epsilon}(t)-x_{\lambda}(t) \|^{2} \\
& +\left(\partial \phi_{\epsilon}\left(x_{\epsilon}(t)\right)-\partial \phi_{\lambda}\left(x_{\lambda}(t)\right), x_{\epsilon}(t)-x_{\lambda}(t)\right) \\
\leq & \left(f\left(x_{\epsilon}(t)\right)-f\left(x_{\lambda}(t)\right), x_{\epsilon}(t)-x_{\lambda}(t)\right) .
\end{aligned}
$$

Since

$$
\begin{aligned}
& \left(f\left(x_{\epsilon}(t)\right)-f\left(x_{\lambda}(t)\right), x_{\epsilon}(t)-x_{\lambda}(t)\right) \\
\leq & \left\|f\left(x_{\epsilon}(t)\right)-f\left(x_{\lambda}(t)\right)\right\|\left\|_{*}\right\| x_{\epsilon}(t)-x_{\lambda}(t) \| \\
\leq & \frac{1}{2 c}\left\|f\left(x_{\epsilon}(t)\right)-f\left(x_{\lambda}(t)\right)\right\|_{*}^{2}+\frac{c}{2}\left\|x_{\epsilon}(t)-x_{\lambda}(t)\right\|^{2}
\end{aligned}
$$

for every real number $c$, so if we choose a constant $c$ satisfying $c_{0}-c / 2>0$, then by integrating (3.4) over $[0, T]$ we have

$$
\begin{aligned}
& \frac{1}{2}\left|x_{\epsilon}(t)-x_{\lambda}(t)\right|^{2}+\left(c_{0}-c / 2\right) \int_{0}^{T}|| x_{\epsilon}(t)-x_{\lambda}(t) \|^{2} \\
\leq & \int_{0}^{T}\left(\partial \phi_{\epsilon}\left(x_{\epsilon}(t)\right)-\partial \phi_{\lambda}\left(x_{\lambda}(t)\right), \lambda \partial \phi_{\lambda}\left(x_{\lambda}(t)-\epsilon \partial \phi_{\epsilon}\left(x_{\epsilon}(t)\right)\right.\right. \\
& +\frac{1}{2 c} \int_{0}^{T}\left|x_{\epsilon}(t)-x_{\lambda}(t)\right|^{2}
\end{aligned}
$$

by the monotonicity of $\partial \phi$. Here, we used that

$$
\partial \phi_{\epsilon}\left(x_{\epsilon}(t)\right)=\epsilon^{-1}\left(x_{\epsilon}(t)-(I+\epsilon \partial \phi)^{-1} x_{\epsilon}(t)\right) .
$$

Since $\left|\partial \phi_{\epsilon}(x)\right| \leq\left|(\partial \phi)^{0} x\right|$ for every $x \in D(\partial \phi)$, it follows from (A1) and using Gronwall's inequality that

$$
\left\|x_{\epsilon}-x_{\lambda}\right\|_{C([0, T] ; H) \cap L^{2}(0, T ; V)} \leq C(\epsilon+\lambda), \quad 0<T .
$$

Thus, letting $\lambda \rightarrow 0$, the proof of lemma is complete.

We assume

(F1) $f$ is uniformly bounded: there exists a constant $M_{f}$ such that

$$
|f(t, x)| \leq M_{f}
$$

for all $x \in V$.

In view of Lemma 3.1, if we define the nonlinear operator $\mathcal{F}$ on $L^{2}(0, T ; H)$ by

$$
(\mathcal{F} k)(t)=f\left(t, y_{k}(t)\right)+\partial \phi_{\epsilon}\left(y_{k}(t)\right), \quad k \in L^{2}(0, T ; H),
$$

then $\mathcal{F}$ is a compact mapping from $L^{2}(0, T ; H)$ to itself. Then it holds that

$$
\|\mathcal{F}(k)\| \leq\left(M_{f}+M_{1}\right) \sqrt{T} .
$$

Let

$$
N=\left\{p \in L^{2}(0, T ; H): \int_{0}^{T} S(T-s) p(s) d s=0\right\}
$$


and denote the orthogonal complement of $N$ in $L^{2}(0, T ; H)$ by $N^{\perp}$. We denote the range of the operator $B$ by $H_{B}$.

We need the following assumption:

(B) For each $p \in L^{2}(0, T ; H)$ there exists an element $q \in \bar{H}_{B}$ such that

$$
\int_{0}^{T} S(T-s) p(s) d s=\int_{0}^{T} S(T-s) q(s) d s,
$$

that is, $L^{2}(0, T ; H)=\bar{H}_{B}+N$, where $\bar{H}_{B}$ is the closure of $H_{B}$ in $L^{2}(0, T ; H)$.

For $u \in N^{\perp}$, let $P u$ be the unique minimum norm element of $\{u+N\} \cap \bar{H}_{B}$. Then the proof of Lemma 1 of Naito [9] showed that $P$ is a linear and continuous operator from $N^{\perp}$ to $\bar{H}_{B}$. Let $\tilde{Y}=L^{2}(0, T ; H) / N$ be the quotient space and the norm of a coset $\tilde{u}=u+N \in \tilde{Y}$ be defined of $\|\tilde{u}\|=\inf \{|u+f|: f \in N\}$.

We define by $Q$ the isometric isomorphism from $\tilde{Y}$ onto $N^{\perp}$, that is, $Q \tilde{u}$ is the minimum norm element in $\tilde{u}=\{u+f: f \in N\}$. Let

$$
\tilde{\mathcal{F}} \tilde{u}=\mathcal{F}(P Q \tilde{u})+N
$$

for $\tilde{u} \in \tilde{Y}$. Then we have

$$
\|\tilde{\mathcal{F}}(\tilde{u})\| \leq\left(M_{f}+M_{1}\right) \sqrt{T}
$$

and $\tilde{\mathcal{F}}$ is a compact mapping from $\tilde{Y}$ to itself.

We define the reachable sets for the system (NCE) as follows:

$$
\begin{aligned}
& R_{T}=\left\{x\left(T ; \phi_{\epsilon}, f, u\right): u \in L^{2}(0, T ; U)\right\}, \\
& L_{T}=\left\{x(T ; 0,0, u): u \in L^{2}(0, T ; U)\right\} .
\end{aligned}
$$

If $\overline{R_{T}}=H$ where $\overline{R_{T}}$ is the closure of $R_{T}$ in $H$, then the system (NCE) is called approximately controllable at time $T$.

Theorem 3.3. Let $a(\cdot, \cdot)$ be a symmetric quadratic form satisfying $(2.2)$ and let us assume the hypotheses (A1), (F1) and (B). Then we have $L_{T} \subset \overline{R_{T}}$. Therefore, if the linear system (NCE) with nonlinear terms $f+\partial \phi \equiv 0$ is approximately controllable, then so is the nonlinear system (NCE).

Proof. We follow the proof of Theorem 1 of Naito [9]. Actually we show that $L_{T} \subset{\overline{R_{T}}}^{V}$, where ${\overline{R_{T}}}^{V}$ is the closure of $R_{T}$ in $V$. Let

$$
\eta=\int_{0}^{T} S(T-s) B v(s) d s \in L_{T}
$$

We will show that there exists $w$ such that

$$
\eta=x_{\epsilon}(T ; \phi, f, w) \text {. }
$$

Let $r$ be a positive number such that

$$
v \in U_{r}=\left\{u \in L^{2}(0, T ; U):\|u\|_{L^{2}(0, T ; U)}<r\right\} .
$$

Put $z=B v$ and $r_{1}=\|B\| r$. Then it follows that

$$
\tilde{z}=z+N \in V_{r_{1}}=\left\{\tilde{x} \in \tilde{Y}:\|\tilde{x}\|_{\tilde{Y}}<r_{1}\right\} .
$$


Take a constant $d>0$ such that

$$
\left(M_{f}+M_{1}\right) \sqrt{T}+r_{1}<d .
$$

Let us consider the equation

$$
\tilde{z}=\lambda \tilde{\mathcal{F}} \tilde{u}+\tilde{u}, \quad 0 \leq \lambda \leq 1 .
$$

Let $u$ be the solution of (3.6). Since $\tilde{z} \in V_{d}$ and from (3.5)

$$
\begin{aligned}
\|\tilde{u}\| & \leq\|\tilde{z}\|+\|\tilde{\mathcal{F}} \tilde{u}\| \\
& \leq r_{1}+\left(M_{f}+M_{1}\right) \sqrt{T}<d
\end{aligned}
$$

it follows that $\tilde{u} \notin \partial V_{d}$ where $\partial V_{d}$ stands for the boundary of $V_{d}$. Thus by the homotopy property of degree theory, there exists $\tilde{u} \in \tilde{Y}$ such that

$$
\tilde{z}=\tilde{\mathcal{F}} \tilde{u}+\tilde{u} .
$$

Put $u=Q \tilde{u}$ and $u_{B}=P Q \tilde{u}$. Then we have that $u_{B}=P u$ and $u-u_{B}=$ $u-P u \in N$. Hence

$$
\tilde{z}=\mathcal{F}\left(u_{B}\right)+u+N=\mathcal{F}\left(u_{B}\right)+u_{B}+N .
$$

Therefore,

$$
\begin{aligned}
\eta & =\int_{0}^{T} S(T-s)\left(\mathcal{F}\left(u_{B}\right)(s)+u_{B}(s)\right) d s \\
& =\int_{0}^{T} S(T-s)\left(f\left(s, y_{u_{B}}(s)\right)+\partial \phi_{\epsilon}\left(y_{u_{B}}(s)\right)+u_{B}(s)\right) d s .
\end{aligned}
$$

Since $u_{B} \in \bar{H}_{B}$, there exists a sequence $\left\{v_{n}\right\} \in L^{2}(0, T ; U)$ such that $B v_{n} \mapsto u_{B}$ in $L^{2}(0, T ; H)$. Then by Theorem 2.3 we have that $x_{\epsilon}\left(\cdot ; \phi, f, v_{n}\right) \mapsto y_{u_{B}}$ in $L^{2}\left(0, T ; D\left(A_{0}\right)\right) \cap W^{1,2}(0, T ; H)$, and hence $x_{\epsilon}\left(T ; \phi, f, v_{n}\right) \mapsto y_{u_{B}}(T)=\eta$ in $V$. Thus from Lemma 3.1 it follows that $\eta \in{\overline{R_{T}}}^{V}$.

Remark 3.4. Under the hypothesis (B), we know that $\overline{R_{T}}=H$. In fact, let $\eta \in D(A)$, then putting $p(s)=(\eta+s A \eta) / T$, it holds that

$$
\eta=\int_{0}^{T} S(T-s) p(s) d s .
$$

From (B), there exists a function $q \in \bar{H}_{B}$ such that

$$
\int_{0}^{T} S(T-s) p(s) d s=\int_{0}^{T} S(T-s) q(s) d s .
$$

By the definition of closure of range of the controller $B$, we can show that there exists a control function $v \in L^{2}(0, T ; U)$ such that

$$
\left|\eta-\int_{0}^{T} S(T-s)(B v)(s) d s\right|<\epsilon \quad \text { for any } \epsilon>0 .
$$




\section{References}

[1] J. P. Aubin, Un thèoréme de compasité, C. R. Acad. Sci. 256 (1963), 5042-5044.

[2] V. Barbu, Nonlinear Semigroups and Differential Equations in Banach space, Nordhoff Leiden, Netherland, 1976.

[3] - Analysis and Control of Nonlinear Infinite Dimensional Systems, Academic Press Limited, 1993.

[4] G. Di Blasio, K. Kunisch, and E. Sinestrari, $L^{2}$-regularity for parabolic partial integrodifferential equations with delay in the highest-order derivatives, J. Math. Anal. Appl. 102 (1984), no. 1, 38-57.

[5] P. L. Butzer and H. Berens, Semi-Groups of Operators and Approximation, Springerverlag, Belin-Heidelberg-Newyork, 1967.

[6] J. M. Jeong, Y. C. Kwun, and J. Y. Park, Approximate controllability for semilinear retarded functional differential equations, J. Dynam. Control Systems 5 (1999), no. 3, 329-346.

[7] J. M. Jeong and H. H. Roh, Approximate controllability for semilinear retarded systems, J. Math. Anal. Appl. 321 (2006), no. 2, 961-975.

[8] J. L. Lions and E. Magenes, Problèma aux limites non homogènes et applications, vol. 3, Dunod, Paris, 1968.

[9] K. Naito, Controllability of semilinear control systems dominated by the linear part, SIAM J. Control Optim. 25 (1987), no. 3, 715-722.

[10] H. Tanabe, Equations of Evolution, Pitman-London, 1979.

[11] H. Triebel, Interpolation Theory, Function Spaces, Differential Operators, NorthHolland, 1978.

Jong YeOUl PARK

Department of Mathematics

Pusan National University

BusAn 609-735, KoreA

E-mail address: jyepark@pusan.ac.kr

Jin-Mun JEONG

Department of Applied Mathematics

Pukyong National University

BusAn 608-737, KoreA

E-mail address: jmjeong@pknu.ac.kr

Hyun-HeE RHo

Department of Mathematics

Pukyong National University

Busan 609-737, Korea

E-mail address: hhn9486@hanmail.net 